\title{
Corela
}

Cognition, représentation, langage

HS-8 | 2010

L'interpellation

\section{Faustine, plena Bassus ibat in reda. L'apostrophe latine du nom propre}

\section{Perrine Vedrenne}

\section{(2) OpenEdition}

1 Journals

Édition électronique

URL : https://journals.openedition.org/corela/1826

DOI : $10.4000 /$ corela.1826

ISSN : 1638-573X

Éditeur

Université de Poitiers

\section{Référence électronique}

Perrine Vedrenne, «Faustine, plena Bassus ibat in reda. L'apostrophe latine du nom propre », Corela [En ligne], HS-8 | 2010, mis en ligne le 24 novembre 2010, consulté le 28 juin 2022. URL : http:// journals.openedition.org/corela/1826 ; DOI : https://doi.org/10.4000/corela.1826

Ce document a été généré automatiquement le 29 septembre 2020

\section{(c) (i) (2)(2)}

Corela - cognition, représentation, langage est mis à disposition selon les termes de la licence Creative Commons Attribution - Pas d'Utilisation Commerciale - Partage dans les Mêmes Conditions 4.0 International. 


\title{
Faustine, plena Bassus ibat in reda. L'apostrophe latine du nom propre
}

\author{
Perrine Vedrenne
}

1 Martial a fait de l'épigramme latine un genre codifié, dont la pointe est un constituant généralement considéré comme essentiel. Mais, plus même que par la pointe, cette forme brève se caractérise par un usage quasi constant de l'interpellation, ce dont témoignent les apostrophes au vocatif.

2 Plus de 4 textes sur 5 dans les 12 livres de ce corpus contiennent au moins une occurrence de nom propre personnel en apostrophe ${ }^{1}$. Quel que soit le genre examiné, en latin du moins, on sait que les apostrophes sont en grande majorité constituées de noms propres

3 La fréquence de l'apostrophe dans les épigrammes de Martial ne semble pas anecdotique, et Pierre Laurens ne s'y est pas trompé, qui voit dans l'emploi de l'apostrophe une «constante stylistique» (1989: 264). Ce spécialiste du genre épigrammatique cerne principalement les effets d'allègement et de rythme que suscite le procédé, en insistant sur sa répétition, et sur son rôle de structuration.

4 Mais nous laisserons de côté la valeur stylistique de l'apostrophe, pour interroger spécifiquement son rôle énonciatif dans le genre discursif en question. Tout est en effet parti d'une question, qui donne son titre au présent article: Quelle différence le locuteur introduit-il dans un énoncé comme Bassus ibat in plena reda, en le réalisant sous la forme précise «Faustine, Bassus ibat in plena reda ${ }^{2}$ »? Dans une situation où le locuteur interpelle un allocutaire et prédique quelque chose d'un tiers, où donc situer, au niveau énonciatif, l'allocutaire interpellé ? En quoi cette modalité particulière est-elle susceptible d'éclairer la définition même de l'apostrophe?

5 Le plus souvent, allocutaire et personne sujet de l'énoncé coïncident, le locuteur parlant à l'autre de cet autre même. L'épigramme est alors tout entière dédiée à cet échange entre le locuteur-poète et son allocutaire, volontiers cible de ses railleries. Au-delà des marques linguistiques de $2^{\mathrm{e}}$ personne, suffisantes pour marquer dans l'énoncé la présence du destinataire, l'apostrophe du nom propre choisi spécifie explicitement son identité : 
Quae te causa trahit uel quae fiducia Romam, / Sexte? Quid aut speras aut petis inde? Refer. / "Causas" inquis "agam Cicerone disertior ipso / atque erit in triplici par mihi nemo foro."/ Egit Atestinus causas et Ciuis - utrumque / noras - ; sed neutri pensio tota fuit. / "Si nihil hinc ueniet, pangentur carmina nobis : / audieris, dices esse Maronis opus." / Insanis : omnes gelidis quicumque lacernis / sunt ibi, Nasones Vergiliosque uides. / "Atria magna colam." Vix tres aut quattuor ista / res lauit; pallet cetera turba fame. / "Quid faciam? suade : nam certum est uiuere Romae." / Si bonus es, casu uiuere, Sexte, potes. (III, 83)

"Quel motif, ou plutôt quelle outrecuidance, te conduit à Rome, Sextus? Qu'en attends-tu, ou qu'y viens-tu chercher ? Réponds. - "Je plaiderai, dis-tu, avec plus d'éloquence que Cicéron lui-même, et je n'aurai pas de rival sur aucun des trois forums." - Atestinus a plaidé, Civis aussi (tu les connais tous les deux) : mais ni l'un ni l'autre n'a pu y gagner de quoi payer son loyer. - "Si rien ne nous échoit de ce côté, nous composerons des vers. Quand tu les entendras, tu diras: "Cela, c'est du Virgile!" - Tu perds la tête : tous ceux que tu vois par là grelotter dans leurs manteaux râpés, ce sont autant d'Ovides et de Virgiles. - "Je fréquenterai l'atrium des grandes maisons." - C'est à peine si trois ou quatre malheureux ont trouvé ainsi à se nourrir: tous les autres, le teint livide, meurent de faim. - "Que faire? Donne-moi un conseil, car je suis bien décidé à vivre à Rome." - Si tu es honnête, tu peux y vivre, Sextus, par chance. $»^{3}$

L'apostrophe de départ (Sexte) permet au locuteur de viser un allocutaire avec qui il partage un ensemble de données contextuelles. Il peut alors mentionner des connaissances communes (utrumque noras, v. 5-6), et se référer à une situation d'énonciation orientée en direction de son allocutaire, lequel assiste avec lui au même spectacle (sunt ibi ; Nasones Vergiliosque uides, v. 10); il peut de même s'inscrire dans un dialogue in praesentia (" "Causas" inquis "agam Cicerone disertior ipso" », v. 3).

On considère habituellement que c'est là le rôle dévolu à l'apostrophe : établir une intercommunication, constituer un appel au dialogue. Mais sélectionner un destinataire, c'est en l'occurrence sélectionner conjointement un cadre de dialogue précis, et lui conférer une singularité telle qu'il s'affranchit de tous les autres, par delà la barrière que constitue la fixation matérielle du texte écrit.

8 Dans l'exemple suivant, on voit ainsi le locuteur s'adresser à Rufus en utilisant des verbes au parfait. Or ceux-ci concernent l'envoi, supposément déjà accompli, du livre II des $E$ ́ pigrammes audit Rufus. En d'autres termes, le texte lui-même (qui se trouve être le dernier $\mathrm{du}$ recueil) anticipe un acte qui ne peut logiquement advenir qu'après sa propre (re)production :

Cursorem sexta tibi, Rufe, remisimus hora / carmina quem madidum nostra tulisse reor: / imbribus inmodicis caelum nam forte ruebat. / Non aliter mitti debuit iste liber. (II, 100)

«Je t'ai dépêché mon coureur, Rufus, à la sixième heure et j'ai lieu de croire qu'il était trempé jusqu'aux os quand il t'a remis mes poèmes : car il se trouvait que le ciel s'effondrait en averses démesurées. Il ne convenait pas que mon livre te fût expédié par un autre temps. »

Le cadre figuratif ${ }^{4}$ de l'énonciation épigrammatique, où domine, hypertrophiée, l'instance de l'interpellé, supplante les autres cadres (temporels et intersubjectifs), et autorise une prégnance absolue de la situation d'énonciation qu'il instaure. Se construit donc par ce biais une fiction pragmatique où, à chaque énoncé, n'est plus affecté qu'un seul destinataire, dont la place apparaît saturée.

$10 \mathrm{Au}$ demeurant, cette coïncidence entre l'apostrophé et le sujet de l'épigramme n'est pas systématique. Il se tisse en effet, çà et là, une relation ternaire où le scripteur dissocie la 
personne interpellée et un tiers tout autre. P. Laurens (1989 : 243) parle d'épigramme in aliquem pour le premier cas, et d'épigramme ad aliquem de aliquo pour le second, l'une et l'autre constituant les "deux grandes modalités ", «les deux formes dominantes » du genre. Eu égard à leur fréquence d'emploi, on pourrait néanmoins distinguer trois types d'agencement :

11 - les noms propres au vocatif, associés à une marque de $2^{\mathrm{e}}$ personne dans la phrase, qui constituent la majorité des occurrences anthroponymiques (épigramme in aliquem):

Nulli, Thai, negas; sed si te non pudet istud, / hoc saltem pudeat, Thai, negare nihil. $(\mathrm{IV}, 12)$

«Tu ne te refuses à personne, Thaïs; mais si tu n'en rougis point, rougis du moins, Thaïs, de ne rien refuser du tout. »

12 - les noms propres sujets d'un verbe à la $3^{\mathrm{e}}$ personne (épigramme de aliquo), qui ne sont donc pas mis en apostrophe:

Thais habet nigros, niueos Laecania dentes. / Quae ratio est? Emptos haec habet, illa suo. $(\mathrm{V}, 43)$

«Thaïs a les dents noires, Laecania les a blanches comme la neige. Quelle en est la raison? C'est que l'une a acheté les siennes, tandis que l'autre les tient de la nature. »

13 - et ce qu'on pourrait nommer des épigrammes « mixtes ", in aliquem de aliquo, où un nom propre au vocatif est contigu à un nom propre sujet d'un verbe à la $3^{e}$ personne. C'est à ces dernières que je m'intéresserai désormais :

Litigat et podagra Diodorus, Flacce, laborat. / Sed nil patrono porrigit: haec cheragra est. (I, 98)

«Diodorus va en justice, Flaccus, et il a la goutte aux pieds. Mais il ne donne rien à son avocat : c'est avoir la goutte aux mains. »

Quintum pro Decimo, pro Crasso, Regule, Macrum / ante salutabat rhetor Apollodotus: / nunc utrumque suo resalutat nomine. Quantum / cura laborque potest! Scripsit et edidicit. (V, 21)

«Jadis, Régulus, le rhéteur Apollodotus saluait Decimus du nom de Quintus, Crassus du nom de Macer : à présent, il leur rend son salut à tous deux sous leur nom véritable. Ô miracle de l'application et du travail! Il a couché leurs noms par écrit et les a appris par cœur! »

Esset, Castrice, cum mali coloris, / uersus scribere coepit Oppianus. (VII, 4)

"A la vue de son teint livide, voici, Castricus, qu'Oppianus s'est mis à faire des vers. » Vindemiarum non ubique proventus / cessavit, Ovidi ; pluvia profuit grandis. / centum Coranus amphoras aquae fecit. (IX, 98)

«Le produit des vendanges n'a pas fait défaut partout, Ovidius : les grandes pluies ont rendu service. Coranus a recueilli cent amphores d'eau. $»^{5}$

14 Alors même que les anthroponymes sont très souvent repris d'une épigramme à une autre, on n'en trouve aucun, dans l'entier du corpus martialien, qui s'inscrive conjointement dans ces trois dispositifs. Nombreuses sont les épigrammes où la personne interpellée, qui constitue le motif thématique de l'épigramme, son sujet, est soit un ami de Martial soit un objet de critiques plus ou moins acerbes (en fonction, notamment, de son statut social). Dans les épigrammes mixtes - où le nom propre au vocatif voisine avec un autre nom propre ayant valeur de sujet grammatical-, la personne interpellée a toujours l'entière sympathie de Martial ; à l'inverse, une relation de cet ordre n'apparaît que très exceptionnellement dans les épigrammes de aliquo. Ainsi, l'anthroponyme Faustinus ne figure jamais dans une épigramme où le nom propre n'apparaît qu'en fonction sujet, alors qu'un nom de courtisane, comme Thais, n'est jamais au vocatif dans les épigrammes mixtes. 

l'objet un statut de partenaire, sans impliquer de distinction hiérarchique. La référence dénominative opère bel et bien une discrimination : l'allocutaire des épigrammes mixtes appartient à une sphère sociale restreinte, celle des familiares du locuteur.

ailleurs, la forme vocative du nom propre, lorsque celui-ci est au contact d'une marque de $2^{\mathrm{e}}$ personne, se justifie souvent par la nécessité de fixer la référence à l'allocutaire, en donnant une identité à ce "tu». L'apostrophe peut ainsi suivre ou précéder immédiatement un pronom au datif ou à l'accusatif, un verbe à un mode personnel, un déterminant possessif :

Pron $2^{\mathrm{e}}$ pers. $+\mathrm{Np}$ Voc + Dét possessif $2^{\mathrm{e}}$ pers. :

Natali tibi, Quinte, tuo dare parua uolebam / munera ; (IX, 53 v. 1-2)

«Pour ta naissance, Quintus, je voulais t'offrir un petit présent ; "

Dét poss.+ Pron $2^{\mathrm{e}}$ pers. + Np Voc :

Priuignum non esse tuae te, Galle, nouercae / rumor erat, coniunx dum fuit illa patris. (IV, 16, v. 1-2)

«On disait bien, Gallus, que tu n'étais pas le beau-fils de ta belle-mère, aussi longtemps qu'elle fut l'épouse de ton père ; »

Désinence verbale de $2^{\mathrm{e}}$ pers. + Np Voc:

Crine ruber, niger ore, breuis pede, lumine laesus, / rem magnam praestas, Zoile, si bonus es .(XII, 54)

«Les cheveux rouges, le visage noir, une jambe trop courte, un œil abîmé, tu fais merveille, Zoïle, si tu es un honnête homme. »

Ainsi, dans le premier exemple, l'apostrophe Quinte est insérée entre le pronom au datif tibi, complément de dare, et le déterminant possessif tuo, détaché du noyau nominal natali. Mais dans le corpus restreint qui nous occupe, celui des épigrammes mixtes, l'apostrophe apparait également au contact immédiat d'un nom propre associé à un verbe à la $3^{\mathrm{e}}$ personne, et au contact d'une marque de $1^{\text {ère }}$ personne. Les deux s'entrecroisent souvent, en particulier avec un possessif de $1^{1 \text { èr }}$ personne déterminant le nom propre sujet :

$\mathrm{Np} \mathrm{Suj} \mathrm{+} \mathrm{Np} \mathrm{Voc.} \mathrm{+} \mathrm{Dét} \mathrm{poss.} \mathrm{nominatif} \mathrm{:}$

Sardonychas, zmaragdos, adamantas, iaspidas uno / uersat in articulo Stella, Seuere, meus.

(V, 11, v. 1-2)

«Des sardoines, des émeraudes, des diamants, des opales, voilà, Severus, ce que mon cher Stella fait tourner autour d'un seul doigt. »

Pron $1{ }^{\text {ère }}$ pers. + Np Voc. + Np Suj :

Priscus ab Aetnaeis mihi, Flacce, Terentius oris / redditur (VIII, 45, v. 1-2)

"Terentius Priscus m'est rendu, Flaccus, des rivages de l'Etna »

Dét $1^{\text {ère }}$ personne pluriel $+\mathrm{Np}$ Voc $+\mathrm{Np}$ Suj :

Non cenat sine apro noster, Tite, Caecilianus : / bellum conuiuam Caecilianus habet. (VII, 59)

«L'ami Caecilianus ne dîne jamais, Titus, sans un sanglier. Le joli convive pour Caecilianus!»

Dans le premier exemple, l'apostrophe Seuere est insérée entre le nom propre sujet Stella et son déterminant meus. Dans le deuxième, le vocatif Flacce suit le pronom au datif mihi et précède la $2^{\mathrm{e}}$ partie du nom propre (Priscus) Terentius sujet. Mais le déterminant peut aussi être un possessif de $1^{\text {ère }}$ personne pluriel, comme en VII, 59, où l'apostrophe Tite se glisse entre le déterminant noster et Caecilianus.

L'identification que permet l'apostrophe du nom propre s'étend donc à toutes les figures de l'énoncé : le tiers, celui dont il est question, est identifié doublement, par son nom propre et par son lien avec le destinataire. De fait, même si ce lien n'est pas explicité, parler à quelqu'un de quelqu'un d'autre en se contentant de le nommer, c'est présupposer 
que ce dernier appartient à la sphère des connaissances des interlocuteurs. Mieux encore, le locuteur lui-même devient identifiable par le biais de l'apostrophe, en tant qu'il est susceptible de s'adresser à Faustinus, à Flaccus, etc. Il n'est plus, dès lors, celui qui dit « je » mais celui qui dit «tu», lequel «tu» authentifie par contrecoup l'instance même qui le profère.

L'apostrophe n'a pas pour seule vertu de permettre une ou plusieurs identifications, immédiates ou indirectes. Il suffit pour s'en convaincre d'examiner les épigrammes votives, où le nom propre au vocatif voisine avec un nom propre sujet du verbe : ne visant pas non plus un destinataire avec qui un éventuel dialogue pourrait s'engager, le vocatif joue un rôle tactique tout autre. Très codifiée, car relevant d'une pratique rituelle, cette forme d'épigramme s'articule en deux temps : présentation de l'objet offert au dieu, puis expression du vœu lui-même, dont la réalisation attendue résulte donc d'un échange consacré. Équivalent verbal du don matériel, la première partie de l'énoncé se caractérise alors, dans les textes votifs du corpus (sélectionnés selon le critère des épigrammes «mixtes ») par une structure récurrente. En tête viennent toujours le déictique hic/haec/ hoc et le pronom de deuxième personne au datif. Suivent, sans ordre fixe, l'apostrophe au dieu, le verbe, le SN COD et le sujet :

Hos tibi, Phoebe, uouet totos a uertice crines / Encolpos, domini centurionis amor,

/ grata Pudens meriti tulerit cum praemia pili. (I, 31, v. 1-3)

«O Phébus, Encolpos, les délices du centurion Pudens, fait vœu de t'offrir toute la chevelure qui pare sa tête, lorsque son maître aura eu la joie d'obtenir le grade dû à son mérite, de primipilaire. »

Haec tibi pro nato plena dat laetus acerra, / Phoebe, Palatinus munera Parthenius, / ut, qui prima nouo signat quinquennia lustro, / impleat innumeras Burrus Olympiadas. (IV, 45 , v. 1-4)

"Ces offrandes, le secrétaire du Palais, Parthénius, te les présente de bon cœur, Phébus, et à pleine cassolette, en faveur de son fils Burrus dont les premiers cinq ans marquent le début d'un deuxième lustre : il demande que cet enfant compte encore d'innombrables Olympiades. »

21 L'épigramme IV, 45 commence par le déterminant haec à l'accusatif neutre pluriel, détaché du noyau nominal (Haec... munera auv. 2). Cette thématisation (marquée en latin par le détachement et la position initiale) manifeste une précellence de la référence déictique, car le déterminant renvoie à l'objet désigné dans la situation d'énonciation avant même de former un SN avec le nom subséquent. Viennent ensuite le pronom de deuxième personne au datif, complément de dat, dans une colocation caractéristique de ce type de texte; puis le verbe dat, l'apostrophe Phoebe en tête du v. 2, l'accusatif munera («les présents», détaché donc de son déterminant) et enfin le nom propre sujet Parthenius.

Dans ce type de configuration, rien ne vient signaler la présence du locuteur, à part les marques grammaticales de $2^{\mathrm{e}}$ personne, qui ne le font qu'indirectement. S'effaçant au profit de l'allocutaire et du délocuté, le «je » n'est plus que celui qui met en relation le dévot et son dieu, un nécessaire intermédiaire pour convoquer la divinité. On retrouve ici le cas décrit par Guy Serbat (1994: 98) de "phrases où le V[ocatif] n'est pas le vrai destinataire de l'énoncé » : sont rangés dans ce type d'emploi, qui ne saurait s'interpréter comme un appel au dialogue, les invocations à Iuppiter, les di boni, di immortales, hercle, ecastor, edepol.

Dans les épigrammes votives qui nous intéressent, l'apostrophe n'est pas là pour établir un échange de paroles avec la divinité. Il ne s'agit pas non plus de rendre cette dernière 
témoin d'un énoncé qui autrement lui resterait étranger. L'apostrophe convoque la divinité en la nommant, cependant que le pronom personnel tibi lui donne sa place et dans l'acte (destinataire de l'offrande comme du vœu) et, par contrecoup, dans l'énoncé qui en est le reflet (complément de dat). Loin d'être le simple «signe [...] d'un appel au dialogue, d'une intercommunication" (Serbat, 1994: 102), l'apostrophe devient signal d'une interaction : il revient à la divinité convoquée de répondre au don et au vœu, en l'exauçant.

L'idée d'intercommunication, à quoi renvoie censément l'apostrophe, fait donc problème : pourquoi faudrait-il toujours n'envisager l'allocutaire que comme un locuteur potentiel? Au juste, l'usage textuel de l'apostrophe n'implique pas que l'instance interpellée soit amenée à prendre à son tour la parole. On sait du reste que la structure de l'épigramme votive a pu être en partie imitée par Martial dans les épigrammes-épitaphes, qui présentent la même construction initiale.

Hos tibi uicinos, Faustine, Telesphorus hortos / Faenius et breue rus udaque prata tenet. / Condidit hic natae cineres nomenque sacrauit / quod legis Antullae, dignior ipse legi. / Ad Stygias aequum fuerat pater isset ut umbras : / quod quia non licuit, uiuat, ut ossa colat. (I, 114)

«Ce parc, Faustinus, proche de ta demeure, ces quelques champs et ces prés bien arrosés, Faenius Telesphorus en est le possesseur. C'est ici qu'il a enseveli les restes de sa fille et qu'il a consacré ce nom, que tu lis, d'Antula par une épitaphe où il eût convenu que le sien fit plutôt gravé. Il eût été plus juste que le père descendît vers les eaux du Styx : mais puisque la destiné ne l'a pas voulu, qu'il vive pour rendre hommage à ces cendres. "

On retrouve ici le déictique hos en tête (le noyau hortos étant rejeté à la fin du vers), suivi du pronom tibi. A proximité apparaissent Faustine, nom propre en apostrophe, et Telesphorus Faenius, nom propre sujet. Il n'est plus désormais question d'offrande au dieu, mais d'un lieu où repose un défunt proche de la tierce personne. Tibi est dans la dépendance de uicinos, mais le tour n'en rappelle pas moins, à l'évidence, celui des épigrammes votives. La comparaison entre cette épigramme et les précédentes montre que là encore, il ne s'agit pas prioritairement d'entrer en dialogue avec Faustinus, même si la chose est, en l'occurrence, fictivement plausible. Comme dans les épigrammes votives, il n'y a aucune trace explicite (à la $1^{\text {ère }}$ personne) du locuteur, quoique la référence dénominative apparaisse beaucoup moins nette: Faustinus n'est pas un personnage connu, il n'a de réalité, dans le texte, qu'en tant qu'allocutaire de Martial.

Dans certaines épigrammes-épitaphes, toutefois, le locuteur refait surface. Ainsi pleure-t-il la mort d'une petite esclave, dans l'exemple suivant, qui appartient au cycle d'Erotion :

Hanc tibi, Fronto pater, genetrix Flacilla, puellam / oscula commendo deliciasque meas, / paruola ne nigras horrescat Erotion umbras / oraque Tartarei prodigiosa canis. (V, 34, v. 1-4)

« Ô Fronton mon père, ô ma mère Flaccilla, je vous recommande cette fillette, objet de mes baisers et délices, pour que la mignonne Erotion ne tremble pas d'effroi devant les ombres funèbres et les gueules monstrueuses du chien du Tartare. ${ }^{6}$

Si l'inscription patente du locuteur dans l'énoncé différencie cette épigramme des précédentes, on retrouve la colocation initiale hanc tibi , avec ici le pronom de $2^{\mathrm{e}}$ personne du singulier, alors même que l'énoncé contient une double apostrophe. Le pronom acquiert ainsi un caractère presque artificiel, qui pourrait même remettre en cause la prétendument nécessaire coréférence entre marque du destinataire, dans la phrase, et apostrophe, hors phrase. 

mais de convoquer un autre, de le rendre présent à l'énoncé. La parole de l'épigrammatiste, sans cet autre, ne vaut rien, et c'est bien en cela que l'ego de l'épigramme martialienne est celui qui dit, non pas « je », mais « tu ». C'est sans conteste l'altérité qui fonde ici la subjectivité : l'apostrophe inscrit l'autre dans l'énoncé et, ce faisant, lui en fait assumer corollairement la charge ; l'objectif n'est plus de dire quelque chose à quelqu'un, sur lui-même ou sur un tiers, mais de dire avec, de dire quelque chose qui soit conjointement assumé par un autre.

Eu égard à cette mise en partage, le locuteur n'a plus seul la responsabilité exclusive de l'énoncé. Cela est particulièrement sensible dans les épigrammes "mixtes" où l'apostrophe est accompagnée d'un verbe de $2^{\mathrm{e}}$ personne : verbe rogatif essentiellement, mais aussi verbe comme uides, aspicis, cernis ou miraris. Dans tous les cas, il importe de mettre en commun une parole, un spectacle ou une scène. L'espace énonciatif est pluriel.

A cet égard, il est remarquable qu'un grand nombre d'épigrammes « mixtes » comportent une question, non point posée à, mais attribuée à l'allocutaire interpellé :

Quo possit fieri modo, Seuere, / ut uir pessimus omnium Charinus / unam rem bene fecerit, requiris? / Dicam, sed cito. (VII, 34, v. 1-4)

«Comment peut-il se faire, Severus, que le plus grand coquin de la terre, Charinus, ait accompli une bonne action? Tu le demandes? Je vais te le dire, et même en peu de mots. »

Qua factus ratione sit requiris, / Qui numquam futuit, pater Philinus? / Gaditanus, Auite, dicat istud, / qui scribit nihil et tamen poeta est. (X, 102)

«Comment a-t-il fait - tu voudrais le savoir - pour devenir père, ce Philinus qui de sa vie ne fit œuvre d'amour? Certain Gaditain, Avitus, pourrait te le dire, lui qui, sans avoir rien écrit, n'en est pas moins poète. "

Quare non habeat, Fabulle, quaeris / Uxorem Themison? habet sororem. (XII, 20)

«Pourquoi, Fabullus, Themison n'a-t-il pas de femme? Tu veux le savoir : c'est qu'il

a une sœur. »

Cur saepe sicci parva rura Nomenti / Laremque villae sordidum petam, quaeris? / Nec cogitandi, Sparse, nec quiescendi / In urbe locus est pauperi. (XII, 57, v. 1-4)

«Pourquoi ai-je l'habitude de me diriger vers les humbles champs de mon aride Nomentum et vers le foyer rustique de ma villa? Tu veux le savoir? C'est que, Sparsus, la méditation et le repos sont à Rome également interdits à qui n'est pas riche.»

L'interrogation apparait le plus souvent sous la forme d'une proposition interrogative indirecte qui précède le verbe rogatif (quaeris, requiris, rogas) dont elle dépend syntaxiquement; comme si la question était laissée en suspens, et que son attribution fût retardée. C'est ce qu'on peut observer dans le premier exemple (VII, 34), où le verbe requiris est placé après l'interrogative indirecte qui ouvre l'épigramme (Quo possit fieri modo ut uir... bene fecerit). L'apostrophe Seuere est insérée dans cette interrogative, à la charnière de la régissante et de la complétive qui la constituent. Or la force illocutoire des verbes et des énoncés interrogatifs est de fonctionner comme des appels au discours autre, d'engager autrui à devenir à son tour locuteur, à produire un acte verbal (acte ici posé explicitement comme réussi : dicam). Mais ces mêmes énoncés qui appellent un discours autre sont eux-mêmes repris par un autre locuteur. Ainsi, ils ne nous apparaissent qu'à travers ce prisme d'une énonciation "rapportée » et adressée, en retour, au locuteur premier lui-même, par le double biais de la $2^{\mathrm{e}}$ personne verbale et surtout de l'apostrophe. Fonctionnant alors comme une forme d'écho inverse, celle-ci constitue un pur appel au destinataire. 
$\mathrm{Au}$ fil du même énoncé se rencontrent et s'entrecroisent donc deux appels. Dans ce lieu énonciatif de partage, le locuteur est celui qui reprend l'appel de l'autre, et qui donne la parole à ce dernier. L'appel au destinataire n'a pas pour but d'établir une communication mais de montrer que la communication, déjà engagée, est à la charge de cet autre devenu allocutaire. Celui qu'on croyait être interpellé est en fait celui qui interpelle, le scripteur ne faisant que représenter ce discours autre dans son propre texte et interagir, répondre à l'interpellation, par l'écriture même de l'épigramme.

\section{BIBLIOGRAPHIE}

Benveniste, E. (1970) [1974]. «L'appareil formel de l'énonciation » in Problèmes de linguistique générale, 2 (pp. 79-88). Paris : Gallimard.

Izaac, H. J. (1942) [1969]. Martial. Epigrammes (Vols 1-3). Texte établit et traduit par H. J. Izaac. Paris : Les Belles Lettres.

Laurens, P. (1989). L'abeille dans l'ambre. Célébration de l'épigramme de l'époque alexandrine à la fin de la Renaissance. Paris : Les Belles Lettres.

Serbat, G. (1996). Grammaire fondamentale du latin : Tome 6, L'emploi des cas en latin. Volume 1 : nominatif, vocatif, accusatif, génitif, datif. Louvain : Peeters.

Vallat, D. (2003). « Un cas d'onomastique bilingue : les anthroponymes grecs chez Martial ». In R. Oniga (Ed.), Il plurilinguismo nella tradizione letteraria latina (pp. 151-171). Rome : Il Calamo.

Vallat, D. (2008). Onomastique, culture et société dans les Épigrammes de Martial. Bruxelles : Latomus.

Wolff, E. (2009). « Martial et le grec ». In B. Bortolussi, M. Keller, S. Minon \& L. Sznajder (Eds.), Traduire, transposer, transmettre dans l'Antiquité gréco-romaine(pp. 127-133). Paris : Picard.

\section{NOTES}

1. Sur la question des noms propres chez Martial, cf. Vallat (2003 et 2008), ainsi que Wolff (2008).

2. III, 47 : "Bassus passait (, Faustinus,) dans un chariot plein jusqu'aux bords".

3. Les traductions sont celles de H. J. Izaac pour la CUF.

4. Au sens où le définit E. Benveniste (1970: 85) : «Comme forme de discours, l'énonciation pose deux “figures" également nécessaires, l'une source, l'autre but de l'énonciation. "

5. Tous ces exemples ont été retenus pour leur brièveté. Mais la briéveté n'est pas une règle dans l'épigramme martialienne. Ainsi, l'épigramme III, 58 (Baiana nostri uilla, Basse, Faustini), construite en miroir par rapport III, 47 Faustine, plena Bassus ibat in reda, comprend-elle cinquante-et-un vers.

6. Nous modifions la traduction de H. J. Izaac : « Ô Fronton mon père, ô ma mère Flaccilla, je vous recommande cette fillette : elle fit la joie de mes lèvres et de mon cœur! Que la mignonne Erotion ne tremble pas d'effroi devant les ténèbres infernales et la gueule monstrueuse du chien du Tartare!» 
7. On évitera de reprendre le terme d'«intersubjectivité», pour décrire cette relation énonciative, car ce serait gommer la primauté de «tu » sur «je ».

\section{RÉSUMÉS}

La fréquence des apostrophes, dans les Épigrammes de Martial, est étroitement liée aux particularités énonciatives de ce genre littéraire. L'interpellation devient un moyen pour le locuteur de préserver la situation d'énonciation, en sélectionnant un allocutaire défini par son identification nominative. Mais si l'on peut assez aisément définir le rôle discriminant de l'apostrophe du nom propre dans des énoncés à la $2^{\mathrm{e}}$ personne, il en va autrement dans le cadre d'énoncés du type Faustine, Bassus ibat in plena reda, où le nom propre au vocatif est distinct du nom propre d'un tiers sujet de l'énoncé. Il ne se situe pas sur le même plan que le reste de l'énoncé, car une hiérarchie spécifique se met en place: d'une part, le locuteur s'adresse expressément à un allocutaire, d'autre part, il produit un énoncé qui, ne valant que par l'adresse précédente, est entièrement subordonné à l'apostrophe. A l'issue de l'étude de plusieurs types d'épigrammes contenant cette structure - épigrammes votives, épigrammes-épitaphes, épigrammes introduites par une question attribuée à l'interpellé -, il apparaît que l'apostrophe devient la marque de la primauté de l'allocutaire sur le locuteur, lequel n'est plus désormais « celui qui dit je » mais « celui qui dit $t u$ ». Plutôt que les marques de la subjectivité, ce sont celles de l'altérité qu'il convient de chercher dans l'énoncé. L'apostrophe suppose certes une relation tensive entre locuteur et allocutaire, mais elle en modifie aussi la logique puisque le locuteur choisit de s'y effacer au profit de son partenaire.

The high incidence of apostrophes, in Martial's epigrams, is closely related to enunciative features in this literary genre. The addressing becomes a means for the speaker of preserving the speech situation, by selecting an addressee defined by his nominative identification. But if the discriminating role of the apostrophe of proper noun could be quite easily determinated, that goes differently for utterances such as Faustine, Bassus ibat in plena reda, where proper noun in the vocative case is distinct from a third party's proper noun, subject of the utterance. It is, indeed, not located on the same plan that the rest of the utterance, owing to organization into a specific hierarchy: on the one hand, the speaker explicitly talks to an addressee; and on the other hand he delivers a statement that takes on meaning only in relation to the precedent address, and is therefore entirely submitted to the apostrophe. After studying several kinds of epigrams containing this structure - votive epigrams, epigrams-epitaphs, epigrams introduced by a question asked by the addressee -, it appears that apostrophe becomes the mark of the primacy of the addressee over the speaker, who is no more " the one who says $I$ ", but « the one who says you ». Rather than the marks of subjectivity, those of otherness should be looked for in the utterance. The apostrophe, though involving a tensive relationship between speaker and addressee, changes its logic, since the speaker chooses to stay in the background for his partner.

\section{INDEX}

Mots-clés : apostrophe, nom propre, martial, épigramme, allocutaire

Keywords : proper noun, epigram, addressee 
AUTEUR

PERRINE VEDRENNE

Université Paris 10 